\title{
G-protein coupled receptor expression patterns delineate medulloblastoma subgroups
}

\author{
Kelsey L Whittier ${ }^{1,2}$, Erin A Boese ${ }^{1}$, Katherine N Gibson-Corley ${ }^{4}$, Patricia A Kirby ${ }^{4}$, Benjamin W Darbro ${ }^{1}$, Qining Qian', \\ Wendy J Ingram ${ }^{5}$, Thomas Robertson ${ }^{6}$, Marc Remke ${ }^{7}$, Michael D Taylor ${ }^{7}$ and M Sue O'Dorisio ${ }^{1,2,3^{*}}$
}

\begin{abstract}
Background: Medulloblastoma is the most common malignant brain tumor in children. Genetic profiling has identified four principle tumor subgroups; each subgroup is characterized by different initiating mutations, genetic and clinical profiles, and prognoses. The two most well-defined subgroups are caused by overactive signaling in the WNT and SHH mitogenic pathways; less is understood about Groups 3 and 4 medulloblastoma. Identification of tumor subgroup using molecular classification is set to become an important component of medulloblastoma diagnosis and staging, and will likely guide therapeutic options. However, thus far, few druggable targets have emerged. G-protein coupled receptors (GPCRs) possess characteristics that make them ideal targets for molecular imaging and therapeutics; drugs targeting GPCRs account for 30-40\% of all current pharmaceuticals. While expression patterns of many proteins in human medulloblastoma subgroups have been discerned, the expression pattern of GPCRs in medulloblastoma has not been investigated. We hypothesized that analysis of GPCR expression would identify clear subsets of medulloblastoma and suggest distinct GPCRs that might serve as molecular targets for both imaging and therapy.

Results: Our study found that medulloblastoma tumors fall into distinct clusters based solely on GPCR expression patterns. Normal cerebellum clustered separately from the tumor samples. Further, two of the tumor clusters correspond with high fidelity to the WNT and SHH subgroups of medulloblastoma. Distinct over-expressed GPCRs emerge; for example, LGR5 and GPR64 are significantly and uniquely over-expressed in the WNT subgroup of tumors, while PTGER4 is over-expressed in the SHH subgroup. Uniquely under-expressed GPCRs were also observed. Our key findings were independently validated using a large international dataset.

Conclusions: Our results identify GPCRs with potential to act as imaging and therapeutic targets. Elucidating tumorigenic pathways is a secondary benefit to identifying differential GPCR expression patterns in medulloblastoma tumors.
\end{abstract}

Keywords: Medulloblastoma subgroups, G-protein coupled receptors, Therapeutic targets, Imaging targets

\section{Background}

Medulloblastoma is an embryonal tumor of the cerebellum that accounts for $20 \%$ of all pediatric brain tumors and is the most common cause of death from CNS malignancy in children [1]. Furthermore, survivors face a multitude of long-term sequelae secondary to treatment; exposing a developing brain to the cytotoxic therapies

\footnotetext{
* Correspondence: sue-odorisio@uiowa.edu

'Department of Pediatrics, Carver College of Medicine, University of lowa, lowa City, IA 2524 JCP, USA

${ }^{2}$ Neuroscience Graduate Program, Carver College of Medicine, University of lowa, lowa City, IA, USA

Full list of author information is available at the end of the article
}

that are currently offered can result in physical, neurological and intellectual disabilities [1-3]. Historically, medulloblastoma tumors have been treated according to a morphology-based classification system that divides tumors into three principle histopathologic classes: classic, desmoplastic/nodular and large cell/anaplastic (LCA) [4]. The histopathological class informs prognosis, for example tumors displaying LCA morphology generally have the worst prognosis [4]. However, recent advances have utilized genetic profiling to classify medulloblastoma tumors and these techniques have identified medulloblastoma subgroups that differ in both molecular and clinical profiles [5-7]. Various groups have identified 
between four and five potential subgroups [6,8-13]; however, a recent consensus conference determined that evidence supported four distinct subgroups and acknowledged the potential for multiple subtypes within each subgroup [7]. The two most well-defined subgroups are characterized by overactive signaling in the WNT and Sonic hedgehog $(\mathrm{SHH})$ mitogenic pathways. Less is known about the underlying tumorigenesis mechanisms of the remaining two tumor subgroups, Group 3 and Group 4; however, specific genetic aberrations and gene expression characteristics have been found, and epigenetic origins to these tumors have been proposed [14-16]. These four principle medulloblastoma subgroups differ in terms of demographics, predominant histology, likely cell of origin, DNA copy number aberrations and molecular markers $[5,7,17]$. Importantly, the genetic profile has prognostic significance leading investigators to urge translation of genetic classification into clinical therapeutic trials $[7,14,18,19]$. Tumors of the WNT subgroup have the most favorable outcomes and $\mathrm{SHH}$ tumors have an intermediate response to current therapies. The recent development of small molecule inhibitors of the $\mathrm{SHH}$ pathway holds promise for the treatment of these tumor subgroups [20,21]. Group 3 tumors appear to have the worst prognosis using current therapeutic approaches [5]; however, Groups 3 and 4 are less well-characterized, both clinically and genetically, resulting in a lack of potential targets that has hindered the development of novel therapeutic strategies. Identification of tumor subgroup using molecular classification is expected to become an important component of medulloblastoma diagnosis and staging in the near future. Molecular classification will also likely be used to guide therapeutic options, to measure response to therapy and to provide early detection of relapse.

G-protein coupled receptors (GPCRs) are key regulators and points of control in both the $\mathrm{SHH}$ and WNT signal transduction pathways, as well as many other cell signaling mechanisms [22]. GPCRs possess characteristics that make them ideal targets for molecular imaging and therapeutics; including that they are membranebound, their ligands bind with high affinity and specificity, and that the receptor-ligand complex is subsequently endocytosed carrying the ligand into the tumor cell [23]. The utility of targeting GPCRs in medulloblastoma has been demonstrated with the advent of somatostatin receptor targeted imaging and therapy [24,25] and Octreoscans are now able to differentiate medulloblastoma from lowgrade cerebellar tumors and scar tissue [25-28]. Molecularly targeted imaging has the potential to provide in vivo classification, and in vivo measurement of response to treatment as well as early detection of relapse. Furthermore, molecularly targeted chemo- or radiotherapy has the potential to decrease or alleviate long-term toxic effects of external beam radiotherapy.
While the molecular expression patterns of many genes and proteins in medulloblastoma subgroups have been discerned, subgroup-specific GPCR expression patterns have not previously been investigated. A subset of GPCRs appear on commonly used gene chips, such as the Affymetrix U133 chip; however these chips do not allow for the detection of under-expressed genes [29,30]. Our approach, using quantitative GPCR arrays (Taqman), allows for the assessment of both over- and under-expressed GPCRs.

The aim of this study was to discover G-protein coupled receptors that could serve as targets for imaging and therapeutic agents in medulloblastoma, and we have successfully identified potential receptor targets. Elucidating tumorigenic and potentiating mechanisms in medulloblastoma subtypes has been a secondary benefit to our study.

\section{Methods}

Human tumor cohort

Tumors analyzed for GPCR expression consisted of snapfrozen tumor tissues from 41 medulloblastomas, representing primary surgical resection tissue. Normal pediatric cerebellum was used as control tissue. Both specimen types were acquired from the Cooperative Human Tissue Network (Columbus, OH), The Queensland Children's Tumour Bank (Queensland, AUS), The Children's Cancer Research Unit at the Children's Hospital at Westmead (Westmead, AUS), the Knight Cancer Institute Biolibrary at Oregon Health and Sciences University (Portland, OR) and from patients of the University of Iowa Hospitals and Clinics (UIHC) Children's Hospital. Basic clinical data including age and sex were also obtained. The histopathological reports were acquired with the majority of tumor samples and more extensive pathology reports including cytogenetics were available for some patients. UIHC specimens were acquired under an Institutional Review Board (IRB) approval. Specimens acquired from other sources were de-identified and use of these tissues was declared "Not Human Research" by the University of Iowa IRB.

\section{RNA isolation and GPCR expression arrays}

RNA was isolated from snap-frozen tumor tissue using the PerfectPure RNA Tissue Kit (5Prime); the quantity and quality of RNA was evaluated using a Nanodrop 1000 Spectrophotometer (Thermo Fischer Scientific) and an Agilent 2100 Bioanalyzer. RNA of sufficient quality was defined as having an RNA Integrity Number (RIN) of at least 6 on a scale of $1-10$; RINs in the 8-9.5 range were most commonly seen. The High-Capacity Reverse Transcription Kit (Applied Biosystems) was used to convert the isolated RNA to cDNA. The resultant cDNA of each tumor sample was then applied to a TaqMan $\mathrm{Hu}$ man GPCR Array (Applied Biosystems) which contains 380 TaqMan Gene Expression Assays arranged in a 384 
well-plate (four control wells are included). Each GPCR array was subsequently run on a 7900HT Fast Real-Time PCR System (Applied Biosystems) and the resulting data was analyzed using the SDS/Relative Quantification Manager v.1.2 and the DataAssist v.3.0 software packages (both Applied Biosystems).

\section{Statistical analysis}

Statistical calculations were performed by the DataAssist (v3.0; Applied Biosystems) software. Maximum allowable $C_{T}$ value was set at 40.0 and these values were included. The global normalization method was employed [31]. All p-values were adjusted using the BenjaminHochberg False Discovery Rate to correct for multiple testing and the occurrence of false positives. Heat maps are the result of unsupervised hierarchical clustering performed by DataAssist. Distances between tumor samples were calculated for clustering based on the $\Delta C_{T}$ values using Pearson's Correlation; complete linkage was used as the clustering method.

\section{Histology}

Formalin-fixed paraffin embedded (FFPE) tissues were obtained from the previously mentioned tissue banks in the form of $4 \mu \mathrm{m}$ thick sections on slides. These tissues were routinely stained with hematoxylin and eosin (HE) to determine architectural and morphological features, including desmoplasia, nodular formation, and large-cell/ anaplastic features. Dominant histologic category was determined by a neuropathologist.

\section{Immunohistochemistry}

On cases in which FFPE material was available, subgrouping was accomplished following an immunohistochemical method established at St. Jude Children's Research Hospital that uses immunoreactivity patterns to four antibodies ( $\beta$-catenin, YAP1, GAB1 and filamin A) to categorize tumors into the WNT and $\mathrm{SHH}$ subgroups and Non-WNT/SHH tumors [32]. In this study, the $\mathrm{SHH}$ and WNT subgroups, and Non-SHH/WNT tumors were identified via immunoreactivity patterns to two of these markers: $\beta$-catenin (Abcam \#ab16051) and YAP1 (Santa Cruz \#sc-101199).

Antigen unmasking of paraffin sections was performed (citrate buffer, $\mathrm{pH}$ 6) in a decloaker and endogenous peroxidase activity was quenched with $3 \%$ hydrogen peroxide. Sections were incubated with the primary antibody ( $\beta$-catenin at 1:2000; YAP1 at 1:1000) for $60 \mathrm{~min}$ $(\beta$-catenin) or 30 minutes (YAP-1) and then incubated with DAKO Mouse Envision HRP System reagent for 30 minutes for $\beta$-catenin or 15 minutes for YAP1. Slides were developed with DAKO DAB plus for 5 min followed by $\mathrm{DAB}$ Enhancer for 3 minutes before counterstaining with hematoxylin.

\section{Fluorescence in situ hybridization (FISH)}

In cases in which there was sufficient material, FISH to determine $C-M Y C$ and/or $N-M Y C$ amplification was performed. The DNA probes $C-M Y C$ BAP and $N-M Y C /$ CEP2 (Abbott Molecular) were utilized. The sections with $C-M Y C$ copy number gains were sequentially probed with a CEP8 probe to further assess chromosome 8 copy number gains vs specific $C-M Y C$ amplification. The protocols for FFPE slide preparation and hybridization were as per manufacturer's specifications. Briefly, after deparaffinizing, enzyme-pretreating and fixing the sections, the hybridizations were performed on a ThermoBrite (Abbott Molecular) programmed for melt temperature at $85^{\circ} \mathrm{C}$ and time for 2 minute. After overnight hybridization at $37^{\circ} \mathrm{C}$, the slides were washed in $0.4 \mathrm{XSSC} / 0.3 \% \mathrm{NP}-40$ for 2 minutes at $73^{\circ} \mathrm{C}$ and in $2 \mathrm{XSSC} / 0.1 \% \mathrm{NP}-40$ for 1 minute at room temperature. The slides were then counterstained with DAPI. The slides were analyzed and images acquired using CytoVision computerized imaging system (Leica, USA).

\section{Independent correlation of GPCR expression patterns}

Three independent previously published gene expression $[8,10-12]$ datasets were analyzed using the R2 software (http://r2.amc.nl). Expression patterns of LGR5, GPR64, PTGER4, FZD2 and F2R were compared according to the four medulloblastoma subgroups. Differential expression of these candidate genes was assessed using one-way ANOVA. P-values $<0.05$ were considered to be statistically significant.

\section{Results}

\section{GPCR expression patterns}

RNA from 41 human medulloblastoma tumors and four normal human cerebellum specimens were subjected to qPCR analysis of GPCR expression levels. Clusters of medulloblastoma tumors emerged based solely on their GPCR expression patterns (Figure 1; Additional file 1: Figure S1 includes the GPCR loci). Unsupervised hierarchical clustering of all 45 samples revealed varying numbers of groups, depending on the level of association. Two clusters of tissue samples emerged at the lowest level of association: one cluster of 14 tumors designated cluster " $E$ " (Figure $1 \mathrm{~b}$ and Table 1 ) and a second cluster including the remaining tumor 27 samples, as well as the four normal cerebellar controls.

The next level of association split this cluster of 31 specimens (27 tumors and 4 controls) into two further clusters: 1) four tumor samples (cluster " $D$ " in Figure 1b and Tables 1 and 2) four cerebellar control samples plus one tumor sample (Controls B, C, D, E and MB 40). The other 21 tumors could be further divided into three clusters designated " $C$ ” $(\mathrm{n}=10)$, " $B$ " $(\mathrm{n}=4)$, and " $A$ ” ( $(\mathrm{n}=7)$ in Figure $1 \mathrm{~b}$ and Table 1 . One tumor sample associated 


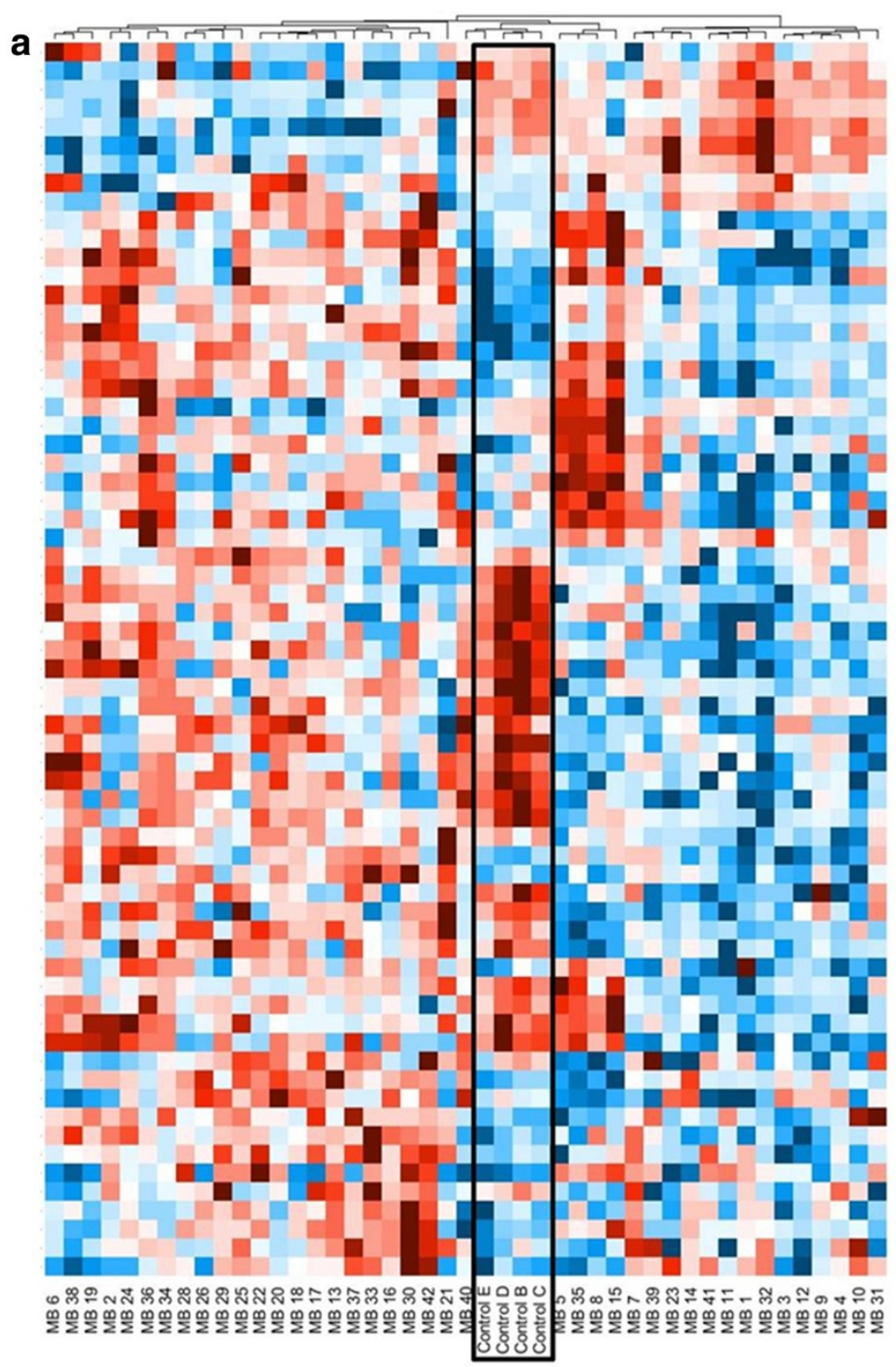

b

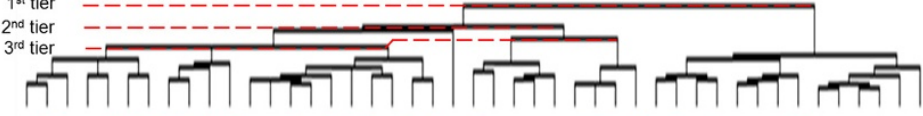

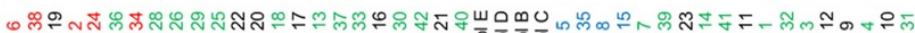

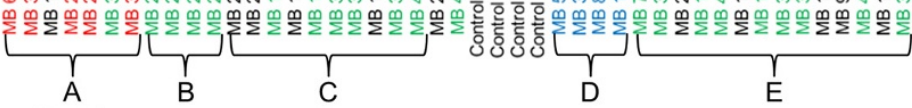

$(\mathrm{SHH})$

(WNT)

Figure 1 GPCR expression patterns delineate distinct groups of medulloblastoma tumors. The heat map represents GPCR expression levels in 41 medulloblastoma tumors compared to normal pediatric cerebella (a). Red color indicates low $C_{T}$ on qPCR, corresponding to high RNA expression; blue is high $C_{T}$ and low RNA expression. Control cerebella (Controls $B, C, D, E$ ) are outlined in the black box. This heat map displays the results of unsupervised hierarchical clustering performed by DataAssist (v3.0; Applied Biosystems) software. Presented in this heat map are those GPCRs that were over- or under-expressed, as compared to control cerebellum, at a $p \leq 0.10$ level. An enlarged image of the clustering stems, as well as the resultant tumor groups is seen in (b). Distances between tumor samples were calculated for clustering based on the $\Delta \mathrm{C}_{T}$ values using Pearson's Correlation; complete linkage was used as the clustering method. Groups (A-E) of medulloblastoma tumors have emerged based solely on their GPCR expression patterns (b). Subsequent immunohistochemical subtyping of the medulloblastoma samples identified tumors of the SHH, WNT and Non-WNT/SHH subgroups (Figure 2, Table 2). The SHH- and WNT-subgroup tumors clustered tightly together on the GPCR expression heat map. Red color indicates tumors identified as belonging to the SHH subgroup, blue indicates WNT subgroup tumors and green indicates tumors identified as Non-WNT/SHH. FFPE slides not available for classification for those medulloblastoma samples in black. 
Table 1 GPCR expression levels by linkage analysis clusters, compared to normal cerebella

\begin{tabular}{|c|c|c|c|c|c|c|c|c|c|c|}
\hline & \multirow{2}{*}{\multicolumn{2}{|c|}{$\begin{array}{c}\text { Cluster "A" } \\
(n=7)\end{array}$}} & \multirow{2}{*}{\multicolumn{2}{|c|}{$\begin{array}{c}\text { Cluster "B" } \\
(n=4)\end{array}$}} & \multirow{2}{*}{\multicolumn{2}{|c|}{$\begin{array}{c}\text { Cluster "C" } \\
(n=10)\end{array}$}} & \multirow{2}{*}{\multicolumn{2}{|c|}{$\begin{array}{c}\text { Cluster “D” } \\
(n=4)\end{array}$}} & \multirow{2}{*}{\multicolumn{2}{|c|}{$\begin{array}{c}\text { Cluster E" } \\
(n=14)\end{array}$}} \\
\hline & & & & & & & & & & \\
\hline & Fold change & $p$-value & Fold change & p-value & Fold change & p-value & Fold change & $p$-value & Fold change & $\mathrm{p}$-value \\
\hline FZD2 & 25 & 0.01 & 27 & 0.04 & 16 & 0.01 & 45 & 0.01 & 4.5 & 0.05 \\
\hline RPLPO & 15 & 0.01 & 7.8 & 0.32 & 9.8 & 0.01 & 28 & 0.01 & 2.9 & 0.19 \\
\hline EDG4 & 25 & 0.01 & 22 & 0.04 & 22 & 0.01 & 20 & 0.03 & 7.8 & 0.04 \\
\hline PTGER4 & 14 & 0.01 & 2.0 & 0.43 & 5.7 & 0.01 & 2.9 & 0.30 & 1.1 & 0.90 \\
\hline GPR126 & 17 & 0.01 & 13 & 0.21 & 15 & 0.01 & 0.67 & 0.76 & 6.1 & 0.08 \\
\hline OR2C3 & 0.035 & 0.01 & 0.026 & 0.29 & 0.0076 & 0.01 & 0.12 & 0.49 & 1.1 & 0.89 \\
\hline FKSG83 & 0.010 & 0.01 & 0.037 & 0.22 & 0.30 & 0.26 & 0.61 & 0.69 & 2.7 & 0.31 \\
\hline$F 2 R$ & 73 & 0.01 & 54 & 0.04 & 55 & 0.02 & 29 & 0.03 & 15 & 0.03 \\
\hline DRD2 & 75 & 0.01 & 24 & 0.10 & 17 & 0.02 & 19 & 0.04 & 4.9 & 0.13 \\
\hline GPR142 & 0.078 & 0.60 & undetectable & $5 . \mathrm{E}-03$ & 0.51 & 0.81 & 1.0 & 0.99 & 0.23 & 0.52 \\
\hline OPRM1 & 0.57 & 0.74 & 0.0028 & 0.22 & 0.0063 & 0.01 & 0.0032 & 0.23 & 0.00050 & 3.E-03 \\
\hline GPR147 & 0.059 & 0.15 & 0.011 & 0.29 & 0.0056 & 0.01 & 0.035 & 0.10 & 0.00050 & 5.E-04 \\
\hline GPR62 & 2.2 & 0.30 & 7.3 & 0.07 & 14 & 0.01 & 10 & 0.15 & 3.1 & 0.08 \\
\hline GPR153 & 3.3 & 0.10 & 8.7 & 0.21 & 8.5 & 0.01 & 0.50 & 0.24 & 3.0 & 0.04 \\
\hline PPYR1 & 0.59 & 0.63 & 0.033 & 0.21 & 0.030 & 0.01 & 0.47 & 0.37 & 1.2 & 0.82 \\
\hline EDG8 & 0.053 & 0.10 & 0.080 & 0.29 & 0.11 & 0.01 & 0.15 & 0.37 & 0.50 & 0.19 \\
\hline GPR160 & 2.4 & 0.19 & 5.9 & 0.22 & 12 & 0.01 & 1.2 & 0.90 & 1.4 & 0.61 \\
\hline SSTR3 & 0.40 & 0.61 & 0.13 & 0.49 & 0.068 & 0.01 & 0.72 & 0.62 & 0.99 & 0.99 \\
\hline GPR10 & 0.15 & 0.19 & 0.11 & 0.38 & 0.082 & 0.01 & 0.23 & 0.20 & 0.58 & 0.36 \\
\hline EDG7 & 0.42 & 0.48 & 0.12 & 0.56 & 0.12 & 0.03 & 0.0079 & 0.01 & 0.032 & 1.E-03 \\
\hline GRM6 & 19 & 0.22 & 326 & 0.29 & 248 & 0.02 & 1539 & 0.01 & 79 & 0.01 \\
\hline CCKBR & 0.28 & 0.60 & 0.12 & 0.29 & 0.039 & 0.03 & 0.0028 & 0.01 & 0.010 & 0.01 \\
\hline OPRK1 & 0.29 & 0.49 & 1.0 & 1.0 & 0.21 & 0.20 & 0.0027 & 0.01 & 0.046 & 0.03 \\
\hline CHRM4 & 19 & 0.55 & 5.1 & 0.23 & 4.8 & 0.11 & 74 & 0.01 & 3.3 & 0.21 \\
\hline OPRD1 & 11.8 & 0.29 & 3.3 & 0.76 & 1.9 & 0.40 & 344 & 0.01 & 0.85 & 0.88 \\
\hline LGR5 & 0.50 & 0.71 & 0.83 & 0.95 & 0.45 & 0.55 & 117 & 0.01 & 0.22 & 0.17 \\
\hline GPR123 & 0.048 & 0.19 & 0.034 & 0.23 & 0.031 & 0.02 & 0.0041 & 0.15 & 0.0016 & 4.E-04 \\
\hline NTSR2 & 0.023 & 0.03 & 0.030 & 0.23 & 0.080 & 0.03 & 0.0030 & 0.04 & 0.0056 & 5.E-04 \\
\hline TRBV5 & 0.083 & 0.14 & 0.077 & 0.22 & 0.10 & 0.08 & 2.0 & 0.79 & 0.0051 & 3.E-03 \\
\hline ADORA1 & 0.83 & 0.91 & 0.28 & 0.32 & 0.14 & 0.14 & 3.0 & 0.33 & 0.019 & 3.E-03 \\
\hline ADRA1A & 0.27 & 0.38 & 0.066 & 0.23 & 0.024 & 0.03 & 0.0082 & 0.19 & 0.0088 & 0.01 \\
\hline GRM4 & 0.13 & 0.30 & 0.11 & 0.32 & 0.037 & 0.06 & 0.0043 & 0.03 & 0.0048 & 0.01 \\
\hline HTR5A & 0.074 & 0.17 & 0.12 & 0.29 & 0.17 & 0.15 & 0.0091 & 0.03 & 0.017 & 0.01 \\
\hline GPR84 & 0.20 & 0.19 & 0.39 & 0.54 & 0.10 & 0.05 & 0.045 & 0.04 & 0.043 & 0.01 \\
\hline GPR39 & 0.11 & 0.20 & 0.0068 & 0.05 & 0.015 & 0.02 & 0.0041 & 0.03 & 0.0058 & 0.01 \\
\hline GPR77 & 1.0 & 0.99 & 0.47 & 0.54 & 0.85 & 0.85 & 0.14 & 0.51 & 0.041 & 0.01 \\
\hline GPR37L1 & 0.049 & 0.19 & 0.057 & 0.22 & 0.080 & 0.11 & 0.0054 & 0.19 & 0.0058 & 0.01 \\
\hline GPR63 & 1.5 & 0.77 & 0.18 & 0.53 & 0.075 & 0.10 & 1.4 & 0.82 & 0.012 & 0.01 \\
\hline GPR75 & 0.48 & 0.43 & 0.15 & 0.21 & 0.22 & 0.11 & 0.13 & 0.06 & 0.029 & 0.01 \\
\hline OR2A4 & 0.51 & 0.50 & 5.7 & 0.22 & 3.7 & 0.08 & 2.1 & 0.51 & 3.3 & 0.01 \\
\hline CD97 & 4.6 & 0.12 & 1.4 & 0.68 & 1.8 & 0.11 & 1.5 & 0.67 & 0.27 & 0.01 \\
\hline
\end{tabular}

* Fold-change $<1$ indicates decreased expression compared to normal cerebellum; all fold-change levels rounded to two significant digits. Significant fold-changes $(p \leq 0.01)$ indicated indicated in bold. 
Table 2 Immunohistochemical groupings of medulloblastoma tumors

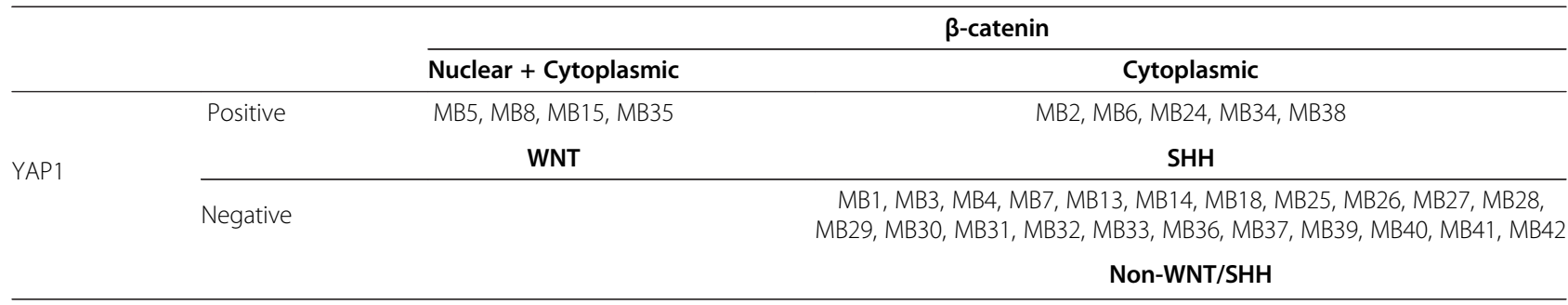

* MB28 and MB37 displayed rare nuclei that were weakly positive for YAP1; both were considered negative for YAP1 immunoreactivity.

alone at this level (MB21). The cerebellar control samples display a GPCR expression profile that is very distinct from each of the five clusters of medulloblastoma tumors (clusters " $A$," " $B$, , " $C$," " $D$ " and " $E$;" Figure 1).

\section{GPCR expression levels in linkage analysis clusters}

The fold-change in expression of GPCRs between tumor and normal tissue was evaluated in the distinct clusters $(A-E)$ of medulloblastoma. Table 1 summarizes the GPCRs that were over- or under-expressed at a significant level $(\mathrm{p} \leq 0.01)$ in one or more clusters compared to normal cerebella. No GPCRs were significantly altered in all five clusters at this significance level.

Among the 380 GPCRs probed, nine GPCRs displayed significantly altered expression in cluster " $A$;" seven were over-expressed, ranging from 14-fold (PTGER4) to 75-fold (DRD2) expression, while two (OR2C3 and FKSG83) were under-expressed, compared to normal cerebella (Table 1).

One GPCR (GPR142) exhibited significantly altered expression in cluster "B;" GPR142 expression was undetectable in this cluster. There were no significant alterations in expression levels of GPR142 in the other clusters, compared with normal cerebella.

Expression of 15 GPCRs was significantly altered in cluster " $C$;" six of these GPCRs were common between clusters " $A$ " and " $C$ " and two other GPCRs were common between clusters " $C$ " and " $E$." In cluster " $C$," over-expression was seen in eight of the GPCRs, ranging from 5.7-fold (PTGER4) to 22-fold (EDG4) expression; under-expression in seven GPCRs ranged from 0.01-fold (OR2C3, OPRM1 and GPR147) to 0.11-fold (EDG8) compared to normal cerebellum (Table 1).

Nine GPCRs displayed significantly altered expression levels in cluster " $D$;" two of these GPCRs were common to both clusters " $A$ " and " $C$ " while three other GPCRs were common to cluster " $E$ " (Table 1). Six of the nine GPCRs with altered expression levels in cluster " $D$ " exhibited over-expression, ranging from 28 -fold (RPLP0) to 1500-fold (GRM6).

Twenty GPCRs had significantly altered expression in cluster " $E$ " (Table 1). Two of these GPCRs were common to cluster " $C$ " and three were common to cluster "D." Of the 20 GPCRs with altered expression levels in cluster "E," only two were over-expressed (GRM6 and OR2A4) while the other 18 were under-expressed, as compared to normal cerebella.

\section{Immunohistochemical analysis and categorization}

Formalin-fixed, paraffin-embedded (FFPE) blocks of tumor tissue were available for thirty of the tumors that had been assayed for GPCR expression levels. Immunoreactivity was determined by two independent University of Iowa pathologists, with any differences being resolved between two readers. Additionally, sections of medulloblastoma tumor samples obtained through the Queensland Children's Tumour Bank (MB30 - MB41) were separately probed for immunoreactivity to the same antibodies at Pathology Queensland (Australia). These sections were read by an independent Pathology Queensland pathologist; therefore, for these samples (MB30- MB41), there are three independent readers. A high level of agreement was observed between the two different laboratories.

Tumors were classified based on immunoreactivity patterns, as shown in Table 2. Immunoreactivity to YAP1 has been shown to differentiate WNT and SHH tumors from Non-WNT/SHH (Groups 3 and 4) tumors [32]. Immunoreactivity to YAP1 was found in nine out of $31 \mathrm{tu}-$ mors (Table 2). Nuclear immunoreactivity to $\beta$-catenin is a well-established method for the identification of WNT driven medulloblastoma tumors [5,32]. Nuclear $\beta$-catenin staining in less than $2 \%$ of tumor nuclei was considered sporadic and these samples were read as negative for nuclear $\beta$-catenin staining [32]. Four tumor samples displayed nuclear $\beta$-catenin staining. All four of these tumors positive for nuclear $\beta$-catenin also displayed YAP1 immunoreactivity, and have therefore been classified as a WNT subtype medulloblastoma (Table 2).

Combining the findings from the immunoreactivity patterns to YAP1 and $\beta$-catenin provides a method of differentiating the WNT, SHH and non-WNT/SHH subgroups of tumors. A combination of YAP1 immunoreactivity and nuclear $\beta$-catenin staining (Figure $2 \mathrm{~d}, \mathrm{~h}$ ) segregated the WNT subgroup $(\mathrm{n}=4 ; 13 \%)$, as shown in Table 2 . Positive YAP1 staining without nuclear $\beta$-catenin staining (Figure 2e, i) indicated the SHH subgroup $(\mathrm{n}=5$; $17 \%)$; non-WNT/SHH subgroups were characterized by a lack of 


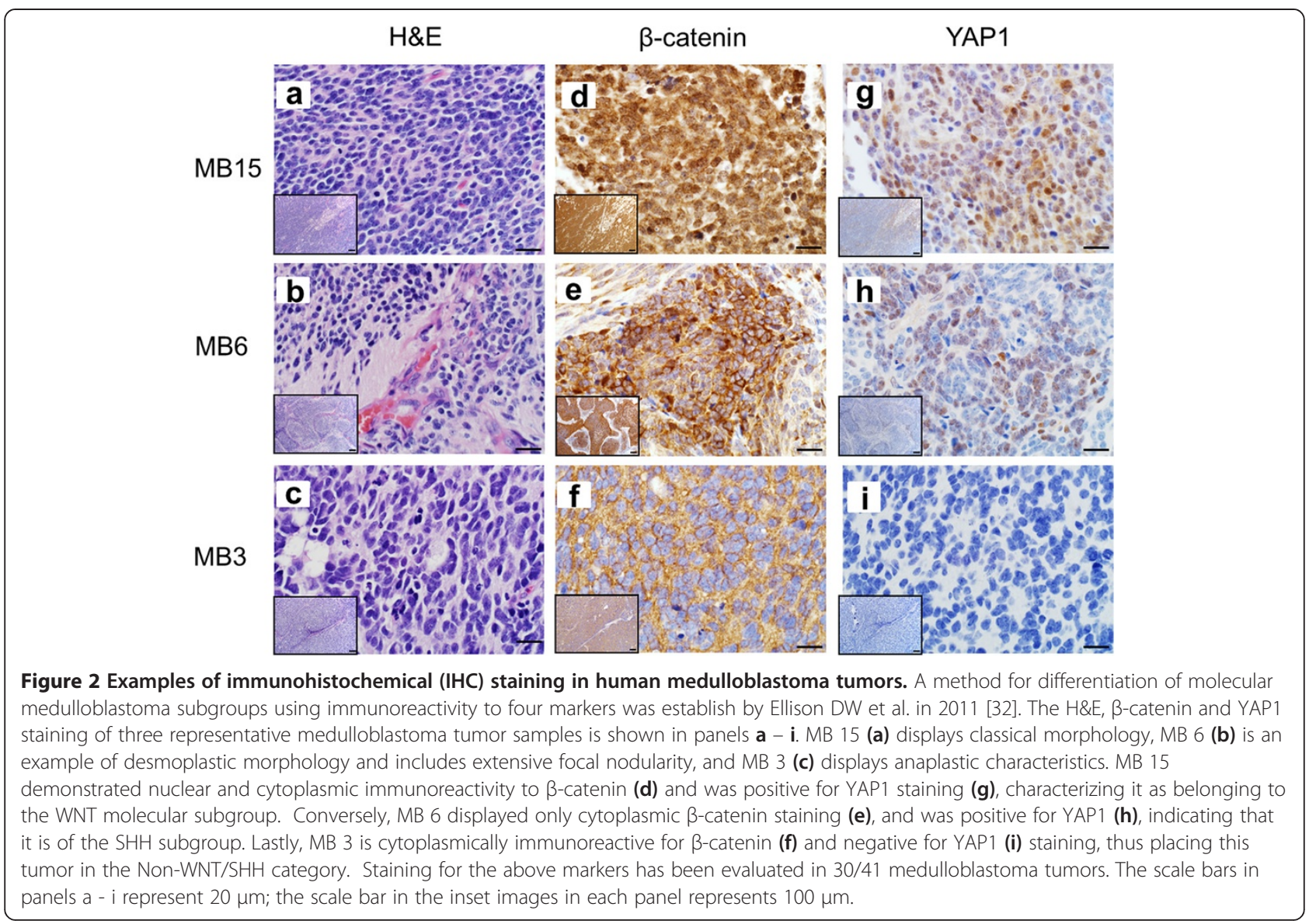

immunoreactivity to both of these antibodies (Figure 2f, i; $\mathrm{n}=22 ; 70 \%)$. The remaining 10 tumors were not classified due to lack of FFPE tissue for the performance of immunohistochemical analysis. Our observed distribution of tumors into the subgroups closely aligns with previously published distributions in larger cohorts [5].

\section{Medulloblastoma subgroups WNT pathway medulloblastomas}

The WNT pathway medulloblastomas $(n=4)$ were identified by a combination of positive YAP1 staining, as well as nuclear and cytoplasmic immunoreactivity to $\beta$-catenin (Figure 2, Table 2). All WNT tumors displayed classic histopathology, characterized by sheets of monomorphic cells with hyperchromatic nuclei and a high nuclear: cytoplasmic ratio (Figure 2) [4]. C-MYC and N-MYC amplification was probed using fluorescent in-situ hybridization (FISH); the N-MYC signal was normal in all four WNT subgroup tumors and no C-MYC amplification was observed, though two WNT tumors displayed increased C-MYC signal due to gains of chromosome 8 (Additional file 2: Table S1). Of the four WNT tumors, $50 \%$ were from male patients, and the age range for all tumors was 5 to 17 years.
The WNT tumors (MB5, MB8, MB15, MB35) tightly clustered together (Figure 1, Table 2) and completely correlate to linkage analysis cluster " $D$ " (Figure 1b). Aside from each other, the WNT tumors were most closely associated with the normal cerebellar control samples. To determine GPCR expression patterns specifically in this subgroup, the WNT tumors were grouped together and the fold-change in expression level of each receptor, as compared to normal controls, was assessed.

The expression levels of 26 GPCRs, out of the 380 receptors probed, were significantly $(\mathrm{p} \leq 0.05)$ altered in WNT tumors compared to expression levels in normal cerebella (Additional file 3: Table S2). Of these 26 GPCRs, 12 were expressed at a significantly lower level than in normal cerebella, while 14 were over-expressed (Additional file 3: Table S2). The levels of under-expression ranged from 0.003-fold (OPRK1) to 0.07-fold (RHO), while the levels of over-expression ranged from 8.8-fold (MRGPRE) to 2200fold (GPR64). Four of the over-expressed GPCRs within the WNT subgroup (FZD2, F2R, EDG4 and RPLP0) were also over-expressed to a significant level within the $\mathrm{SHH}$ subgroup tumors and the Non-WNT/SHH tumors. Five GPCRs (EDG7, CCKBR, GRM4, NTSR2 and GPR84) were significantly under-expressed in the WNT subgroup and 
Non-WNT/SHH tumors, while GRM6 and DRD2 were significantly over-expressed in both groups.

\section{SHH pathway medulloblastomas}

Positive immunoreactivity to YAP1, combined with nonnuclear $\beta$-catenin staining identified tumors of the $\mathrm{SHH}$ subgroup ( $\mathrm{n}=5$; Figure 2, MB6; Table 2). One $\mathrm{SHH}$ subgroup tumor displayed classic histopathology, two tumors exhibited desmoplasia with nodularity and one tumor had anaplastic features. One tumor was classified as having complex histopathology with multiple morphological features. C-MYC and N-MYC FISH data were available for four of the five SHH tumors; all four of these tumors displayed normal C-MYC and N-MYC signals (Additional file 2: Table S1). Within the $\mathrm{SHH}$ subgroup, two patients were female, two were male; age and gender of the patient were unknown for one tumor. Ages of the patients ranged from 1.0 to 2.5 years.

The SHH-subgroup of tumors corresponds to the linkage analysis cluster " $A$ " established by GPCR expression patterns (Figure 1). All five $\mathrm{SHH}$ subgroup tumors (MB2, MB6, MB24, MB34 and MB38) clustered together in a grouping of seven tumor samples; one NonWNT/SHH tumor sample (MB36) and one tumor sample for which FFPE tissue was not available for categorization (MB19) also clustered in this group (Figure 1).

Seven GPCRs in the SHH subgroup displayed significantly altered expression levels when compared to normal cerebellum ( $\leq 0.05$; Additional file 3: Table S2). Six of these altered GPCRs demonstrated over-expression, ranging from 14-fold (RPLP0) to 72-fold (F2R) expression, while one (FKSG83) displayed under-expression (0.01-fold). As discussed above, four GPCRs were also over-expressed to a significant level within all three (WNT, SHH, Non-WNT/SHH) categorized tumor groups (FZD2, RPLP0, EDG4 and F2R). There were no GPCRs that were significantly altered and common to both the SHH and WNT, but not the other subgroups. One GPCR (GPR126) was altered exclusively in the $\mathrm{SHH}$ and the Non-WNT/SHH subgroups and two GPCRs (PTGER4 and FKSG83) were uniquely altered only in the SHH subgroup tumors.

\section{Non-WNT/SHH medulloblastomas}

A lack of YAP1 immunoreactivity in medulloblastoma tumors is indicative of the Non-WNT/SHH subgroup (encompassing both Groups 3 and 4. [32]). Twenty-two tumors were negative for YAP1 (Figure 2, MB3; Table 2). Moreover, all 22 of these tumors also lacked nuclear $\beta$-catenin immunoreactivity (Figure $2 \mathrm{f}$ ), as would be expected for Non-WNT/SHH tumors. Of these 22 tumors, 10 displayed purely classic histopathology, four tumors had classic histopathology along with areas demonstrating anaplastic features, three tumors displayed desmoplastic or nodular/desmoplastic qualities and five tumors exhibited purely large-cell, anaplastic or anaplastic morphology (Additional file 2: Table S1). C-MYC and N-MYC FISH was performed in 18 Non-WNT/SHH subgroup tumors; high-level amplification of C-MYC was seen in three NonWNT/SHH tumors (MB25, MB31 and MB39) while six tumors displayed increased C-MYC copy numbers due to gains of chromosome 8 , and nine tumors had normal C-MYC signal (Additional file 2: Table S1). N-MYC amplification was seen in three Non-WNT/SHH tumors, MB4, MB37 and MB40 (N-MYC/CEP2 ratio of 1.88, 1.8 and 2.3, respectively) and four tumors displayed increased N-MYC signal due to gain of chromosome 2 (Additional file 2: Table S1). Patient characteristics were available for 15 of these tumors; 11 out of 15 tumors were from male patients and patient ages ranged from 1.9 to 8.4 years.

A broad viewing of the GPCR expression patterns heat map shows that the Non- WNT/SHH tumors reside in two large tumor groups, interspersed with tumors for which immunohistochemistry -based subgroup categorization was not possible (Figure 1b). The lowest level of association clusters together 14 tumor samples; this cluster of 14 tumor samples corresponds to the GPCR expression patterns linkage analysis cluster " $E$." Nine of these tumors are of the Non-WNT/SHH subgroup (MB1, MB3, MB4, MB7, MB14, MB31, MB32, MB39, and MB41) and the remaining five tumors in this cluster were uncategorized (MB9, MB10, MB12, MB11 and MB23), as shown in Figure 1b.

Association clusters " $B$ " and " $C$ " encompass the majority of the remaining non-WNT/SHH tumors (Figure 1b). One Non-WNT/SHH group tumor (MB40) clustered with the normal control cerebella samples, and one NonWNT/SHH group tumor (MB36) clustered with the SHH subgroup tumors (Figure 1b). As discussed above, within one of the second tier clusters, three third tier clusters emerged $(n=10,4,7)$; the third tier cluster of four tumors was comprised entirely of Non-WNT/SHH tumors (MB25, MB26, MB28, MB29) and corresponds to linkage analysis cluster " $B$ " (Figure $1 \mathrm{~b}$ and Table 1 ), while the third tier cluster of ten tumors was comprised of six NonWNT/SHH tumors (MB13, MB15, MB30, MB33, MB37 and MB42) and four immunohistochemically uncategorized tumors (MB15, MB17, MB20, MB22) and correlates to cluster " $C$ ". GPCR expression data was not available for one Non-WNT/SHH subgroup tumor (MB27) due to insufficient quality of mRNA.

When comparing GPCR expression levels between the Non-WNT/SHH subgroup tumors and the normal control cerebella, 31 GPCRs displayed significantly altered expression levels ( $\mathrm{p} \leq$ 0.05; Additional file 3: Table S2). Twelve of these GPCRs were over-expressed in NonWNT/SHH tumors compared to control; level of overexpression ranged from 2.8-fold (OR2A4) to 164-fold 
(TACR3). The level of under-expression in 19 of the 31 altered GPCRs ranged from 0.0018-fold (GPR147) to 0.23-fold (EDG8).

\section{Independent correlation of GPCR expression patterns}

Analysis of previously published gene expression profiling data from three independent cohorts of medulloblastoma patients (Toronto, Boston and Heidelberg series $[8,10,11]$, respectively), clearly demonstrates that the GPCR expression patterns observed in our data set hold consistent in the larger cohorts. For example, in two cohorts of subtyped medulloblastoma tumors (188 medulloblastomas and 11 control cerebella [8], and 103 medulloblastomas [10]), both LGR5 and GPR64 were found to be expressed at higher levels in the WNT subgroup tumors, as compared to both normal cerebella and the other three subgroups of tumors (Figure 3a-d); these expression characteristics support our results (Additional file 3: Table S2). Furthermore, we found PTGER4 to be uniquely overexpressed in the $\mathrm{SHH}$ subgroup of medulloblastoma (16-fold, $\mathrm{p}=0.02$; Additional file 3: Table S2) and this finding replicated what was also seen in the Boston and Heidelberg tumor cohorts (Figure 3e, g) $[8,11]$. PTGER4 expression in the $\mathrm{SHH}$ subgroup was increased in comparison to its expression in Group 3 and Group 4 tumors ( $\mathrm{t}$-probabilities $=1.6 \mathrm{e}^{-6}, 2.7 \mathrm{e}^{-8}$, respectively), but not WNT tumors, in the Toronto series of medulloblastoma patients (Figure 3f) [10]. Likewise, F2R and FZD2, both of which were found to be significantly overexpressed in all subgroups of medulloblastoma tumors in our dataset (Additional file 3: Table S2), were also both highly expressed in all tumor groups in previously published larger tumor cohorts (Figure 4) $[8,10,11]$.

\section{Discussion}

The primary goal of this project was to identify G-protein coupled receptors that could serve as targets for imaging and therapeutic agents in medulloblastoma, and this has been successful. GPCR expression patterns also have the potential to elucidate initiating and proliferative mechanisms. The results of this study indicate that GPCR expression patterns delineate five groups of medulloblastoma tumors, two of which correlate with high fidelity to the WNT and the SHH subgroups of medulloblastoma $[5,7,10,14,17,18,32]$. Distinct GPCRs are uniquely overexpressed in the WNT and SHH subgroups, as well as in three other groups of tumors, strongly suggesting that GPCR targets specific to each medulloblastoma subgroup can be identified. Additionally, these data indicate that the unique GPCR expression patterns found may help clarify important mechanistic differences between the groups.

Development of new, or utilization of known ligands to uniquely over-expressed GPCRs offer the potential to provide patient specific information. Subgroup targeted imaging, using radiolabeled GPCR ligands, would offer a non-invasive method to simultaneously diagnose medulloblastoma and characterize molecular subgroups. Importantly, targeted imaging would also afford a sensitive technique for follow-up imaging to determine response to therapy and the presence of metastatic lesions. A benefit in developing imaging agents targeting over-expressed receptors is that the downstream action of the targeted receptor need not correspond to specific tumorigenesis mechanisms. Rather, the viability of an imaging agent is dependent on the following criteria: high affinity and specificity for the target receptor, reliably and highly differential expression of the target receptor between tumor and normal tissue, limited off-target or off-tissue effects, and size constraints. In regard to medulloblastoma, the ability to cross the blood-brain barrier is also crucial. The utility of such a GPCR-targeted imaging modality has already been proven: The Octreoscan, which employs SPECT imaging to detect radiolabeled somatostatin receptor analogues has the ability to differentiate medulloblastoma from low-grade cerebellar tumors and provides an imaging modality to differentiate recurrent medulloblastoma from scar tissue, as well as to localize metastatic lesions [24,25,28]. Positron emission tomography (PET) imaging provides a higher degree of sensitivity than SPECT imaging [28] and somatostatin receptor targeted agents are being adapted for use with PET imaging [28]. Recent evidence has shown that high expression of somatostatin receptors, particularly in non-SHH subgroup tumors, is correlated to an increased survival and may have potential as a prognostic marker [36]. However, previous studies report that somatostatin receptor expression is uniformly high in all medulloblastoma tumors $[37,38]$. Though these studies were performed prior to the advent of medulloblastoma subgrouping, they indicate that somatostatin receptor targeted agents will fail to distinguish between the subgroups of medulloblastoma. Somatostatin receptor, type 2, expression trends towards over-expression in all subgroups, however does not reach significance in our current data set (WNT subgroup: 7.6-fold, $\mathrm{p}=0.24$; SHH subgroup: 5.1-fold, $\mathrm{p}=0.34$; Non-WNT/SHH: 8.1-fold, $\mathrm{p}=0.23$ ).

GPCR-targeted therapeutics, either radioablative or chemically based, have the potential to reduce the need for external beam radiation, and the highly toxic effects associated with cranial spinal radiation treatment [2]. Therapeutically, GPCR antagonists represent the most instinctive approach to counteracting the proliferative signals transduced by some GPCRs within the context of cancer [39]. As such, GPCR antagonists have previously been investigated as potential chemotherapeutic targets in a variety of malignancies [39]. Our data indicate that drugs targeting specific GPCRs may not display the same efficacy in all medulloblastoma tumors, and that subgroup 


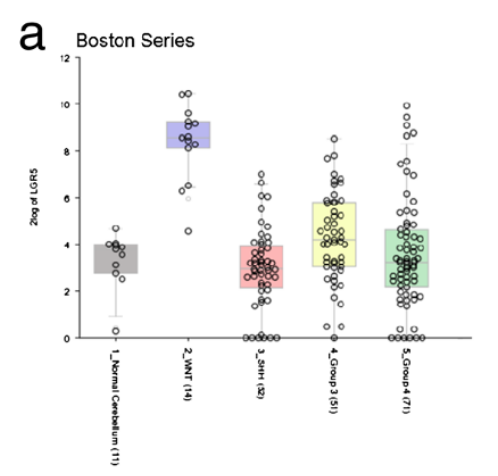

b Toronto Series
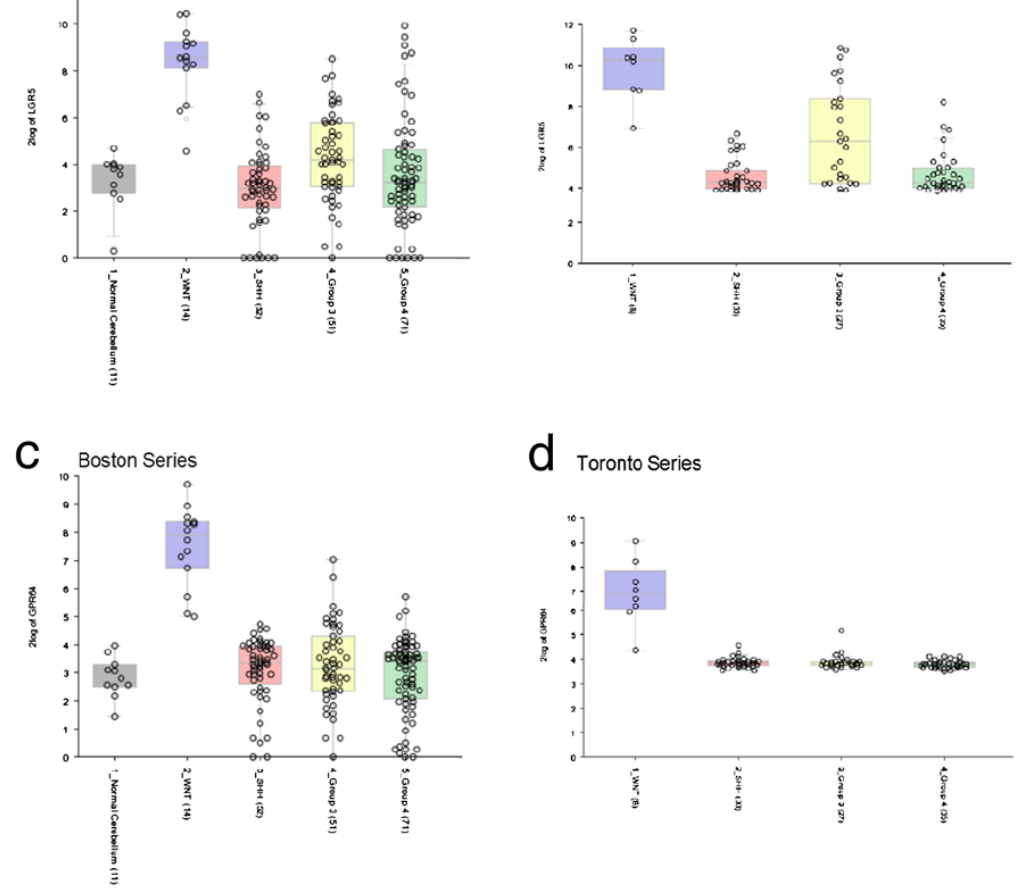

d Toronto Series
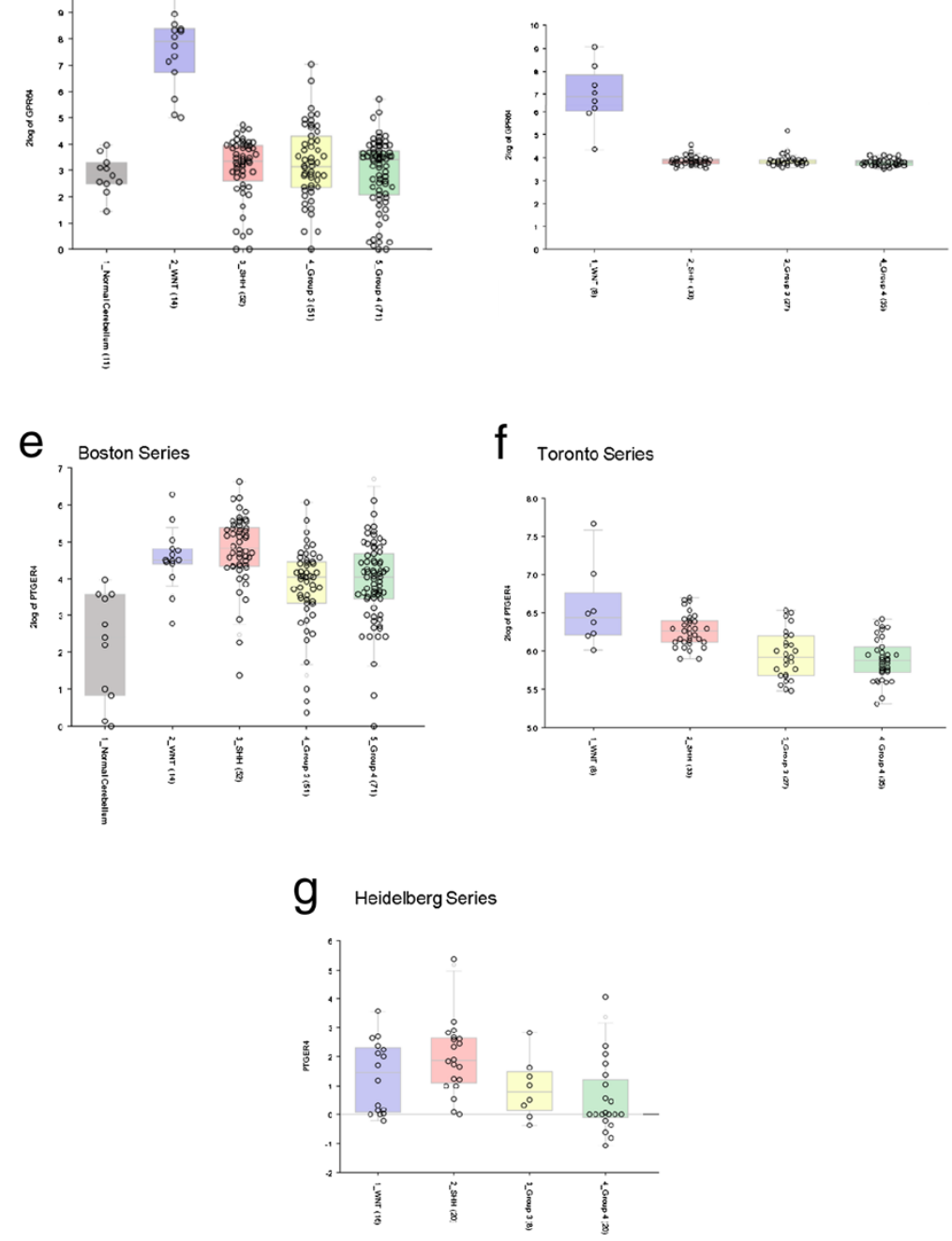

Figure 3 Gene expression profiling data from large cohorts of medulloblastoma tumors qualitatively support our results. In our data set, LGR5 was uniquely over-expressed (120-fold, $p=0.01$ ) in the WNT subgroup of tumors compared to normal cerebellum. Over-expression of LGR5 in WNT tumors has been demonstrated in two previously published sets of gene profiling data $(\mathbf{a}, \mathbf{b})[8,10]$. Our data demonstrate over-expression of GPR64 in the WNT subgroup of tumors (2200-fold, $p=0.04$ ). This aberration was also reported for WNT tumors in the Boston and Toronto series $(\mathbf{c}, \mathbf{d})[8,10]$. PTGER4 was uniquely over-expressed in the SHH subgroup of tumors in our data set $(16-$ fold, $p=0.02)$. This pattern of expression was also observed in the Boston and Heidelberg series $(\mathbf{e}, \mathbf{g})[8,11]$; in the Toronto series (f) $[10]$, the SHH subgroup shows increased PTGER4 expression as compared to Group 3 and Group 4 tumors, but not compared to the WNT subgroup. Blue boxes represent WNT tumors, red boxes represent SHH tumors, yellow boxes represent Group 3 tumors and green boxes represent Group 4 tumors $(\mathbf{a}, \mathbf{b}, \mathbf{c}, \mathbf{d}, \mathbf{e}, \mathbf{f}, \mathbf{g})$. In the Boston series $(\mathbf{a}, \mathbf{c}, \mathbf{e})$, the grey boxes represent normal cerebellar controls. 


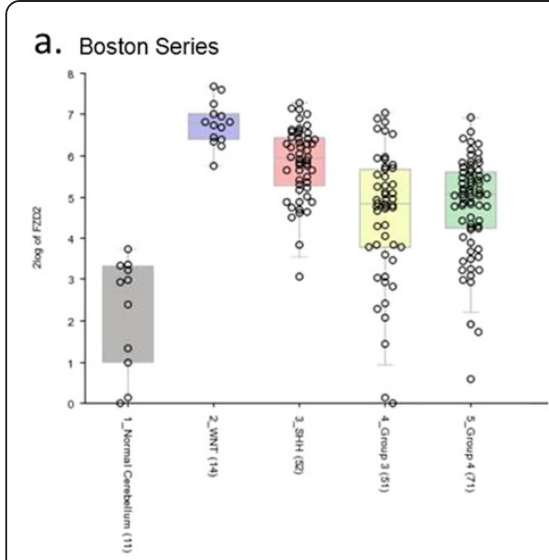

b. Toronto Series

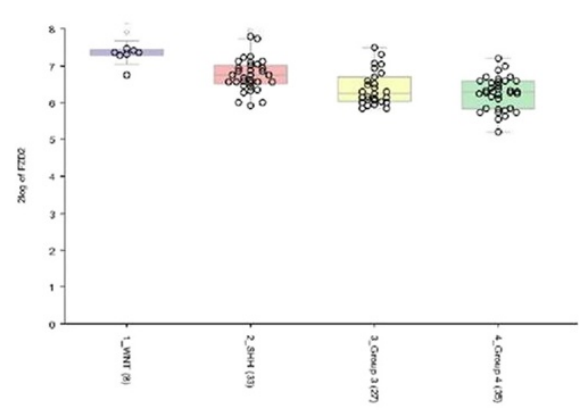

d. Boston Series

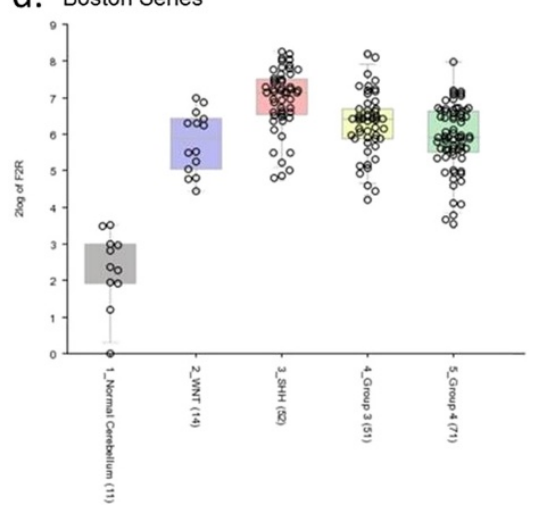

e. Toronto Series

Toronto Series

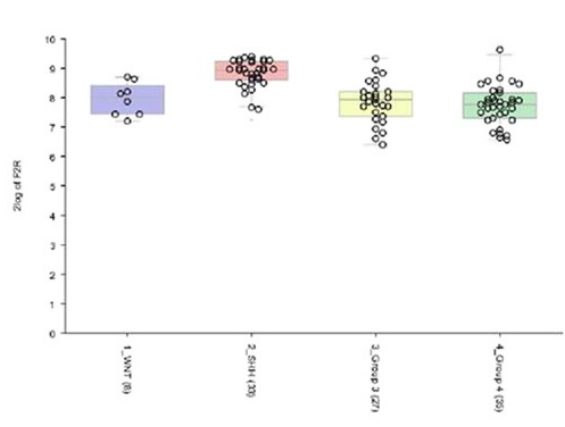

C. Heidelberg Series

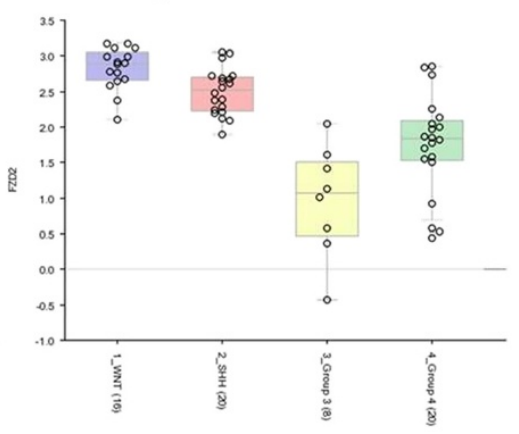

f. Heidelberg Series

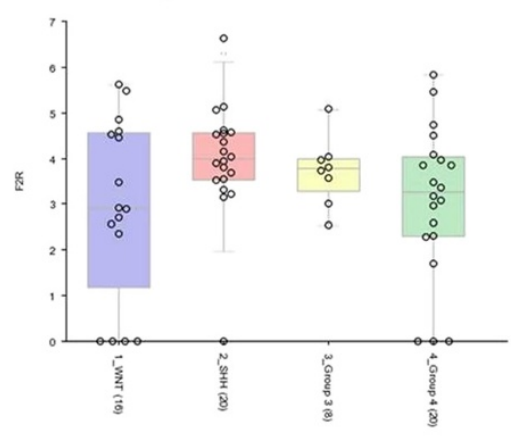

Figure 4 Gene expression profiling data from large cohorts of medulloblastoma tumors qualitatively support our results. Both FZD2 and F2R were significantly over-expressed in all subgroups of medulloblastoma tumors in our cohort (Additional file 3: Table S2). Three previously published sets of gene profiling data $[8,10,11]$ found the same pattern to be true for both FZD2 $(\mathbf{a}, \mathbf{b}, \mathbf{c})$ and F2R (d, e, $\mathbf{f})$. Blue boxes represent WNT tumors, red boxes represent SHH tumors, yellow boxes represent Group 3 tumors and green boxes represent Group 4 tumors (a, b, c, d, e, f). In the Boston series $(\mathbf{a}, \mathbf{d})$, the grey boxes present normal cerebellar controls.

specific GPCR targets would likely result in more beneficial outcomes. GPCR targeted radiotherapy, in which an isotope, such as lutetium-177 or yttrium-90, is attached to a receptor ligand, antibody or other molecule in order to target the radioactivity to the desired GPCR, has been successful in the treatment of neuroendocrine tumors and other malignancies $[40,41]$.

While each of the differentially expressed GPCRs in our data set deserves active investigation into its potential as a target, several candidate receptors are outlined below. Twenty-six GPCRs exhibited significantly altered expression levels in the WNT subgroup tumors; thirteen of these were unique to the WNT subgroup, and eight of these thirteen displayed overexpression, as compared to normal cerebella, and thus are candidate receptors for targeting. Leucine-rich repeat containing G-protein-coupled receptor 5 (LGR5) is significantly over-expressed in the WNT subgroup of tumors ( 120 -fold, $\mathrm{p}=0.01$ ); additionally, our data suggest that it is mildly under-expressed in other groups of medulloblastoma, providing the benefit of being highly differentially expressed not only between WNT tumors and normal cerebella, but also between WNT tumors and other medulloblastoma groups (Additional file 3: Table S2; Figure 3). Furthermore, LGR5 holds the intuitive advantage of being involved in the WNT signaling pathway; LGR5, a known marker of certain adult stem cells, forms a complex with Frizzled/LRP and acts to potentiate the WNT signal [42]. The role of LGR5 in cancer biology has been well-described, especially in the realm of gastrointestinal cancers, where antibodies to LGR5 can be used to identify colorectal cancer stem cells [43,44]. Recently, R-spondins, a class of four large, secreted proteins known to enrich WNT signaling, have been identified as high affinity ligands for LGR5 (and LGR4) [45]. R-spondin proteins hold limited potential as imaging or therapeutic agents in medulloblastoma due to their large size (35 kDa) [46] and likely inability to cross the blood-brain barrier $[47,48]$. However, the emergence of high-throughput screening facilities provides the resources to potentially identify key binding elements of the R-spondin proteins. Our data suggest that the development of small-molecule agents targeting the LGR5 receptor is worthy of attention. 
GPR64 displays a favorable differential expression profile for a WNT subgroup target (2200-fold, p = 0.04; Additional file 3: Table S2, Figure 3). GPR64 is an orphan receptor that belongs to a family of adhesion proteins and is normally highly expressed only in the epididymis; however it has recently been found to be expressed in Ewing's sarcoma (ES), as well as other carcinomas, and represents a marker of invasiveness and metastatic potential in ES [49]. The exact signaling mechanism that follows GPR64 activation is yet unknown and a direct connection between GPR64 and WNT signaling is not readily apparent; however, the development of imaging and radiotherapeutic targets is not dependent on the role of downstream mechanisms in proliferation or apoptosis. Due to its differential expression in medulloblastomas, as well as the fact that it is normally only expressed in the epididymis, GPR64 represents a promising candidate for the development of imaging or radiotherapeutic agents that could be potentially efficacious not only in WNT subgroup medulloblastomas, but also Ewing's sarcoma.

PTGER4 is a GPCR that was uniquely over-expressed (16-fold, $\mathrm{p}=0.02$ ) in the $\mathrm{SHH}$ group of tumors (Additional file 3: Table S2). It was also over-expressed in "Cluster C" GPCR-grouped medulloblastomas (5.66-fold, $\mathrm{p}=0.01$; Table 1), however these tumors fell into the Non-WNT/SHH subgroup and the same pattern of PTGER4 expression was not seen in that subgroup as a whole. PTGER4, or $\mathrm{EP}_{4}$, is a receptor for prostaglandin $\mathrm{E}_{2}$ $\left(\mathrm{PGE}_{2}\right)$. $\mathrm{PGE}_{2}$ has been shown to act as a growth promoting molecule that stimulates proliferation, angiogenesis and invasion [50], and is present at high levels in a variety of malignancies [51,52]. Furthermore, the role of $\mathrm{PGE}_{2}$, and its receptors, has been investigated within the context of medulloblastoma [53]. $\mathrm{PGE}_{2}$ induces medulloblastoma cell proliferation in vitro, while inhibition of $\mathrm{PGE}_{2}$ activity was suppressive both in vitro and in vivo [53]. While Baryawno and colleagues [53] found that $\mathrm{PGE}_{2}$ receptors $\mathrm{EP}_{1-3}$ were most important in stimulating medulloblastoma cell growth, our data suggest that tumor subgrouping may affect $\mathrm{PGE}_{2}$ 's role. Small molecule antagonists to $\mathrm{EP}_{4}$ are currently in development for the treatment of inflammatory pain [54]; $\mathrm{EP}_{4}$ represents a particularly viable therapeutic target, as blockage at this site does not interfere with the production of other important prostanoids, and thus avoids the cardiovascular side effects that can be seen with blockage of this pathway [54]. $\mathrm{EP}_{4}$ represents a viable potential target in medulloblastoma, a possibility that is furthered by the fact that inhibition of the prostaglandin cascade has been shown to enhance the cytotoxic effects of radiotherapy [35] presenting the possibility of synergistic combination therapy. Interestingly, $\mathrm{PGE}_{2}$ has been shown to potentiate the WNT signaling cascade, both in colorectal cancer cells [34], as well as in normal adult hematopoietic stem cells [55], and it was recently found that $\mathrm{PGE}_{2}$ upregulates LGR5 [43]. This finding highlights the important crosstalk between the WNT and SHH signaling cascades [56].

While over-expressed GPCRs provide potential targets, their under-expressed counterparts are equally pertinent when probing unanswered mechanistic questions. GPCRs are responsible for initiating intracellular signaling for multiple pathways; these receptors act at the cell surface to integrate and coordinate diverse communicative stimuli between cells, and converge on shared downstream modulators and effectors. Identifying GPCRs down-regulated in medulloblastoma subgroups may pinpoint receptors critical for growth suppression or inhibition, whose underexpression can lead to, or potentiate, the development of cancer. Less is known about the initiating mechanisms at play in Groups 3 and 4 medulloblastomas [7]; identifying differentially under-expressed GPCRs may help identify additional pathways that contribute to tumorigenesis in these subgroups. MTNR1A, a GPCR for melatonin, is significantly under-expressed only in the Non-WNT/SHH group of medulloblastoma tumors $(0.0065$-fold, $\mathrm{p}=0.02)$. Melatonin has been postulated to be a tumor suppressor gene due to its oncostatic effect in various cancers $[33,57,58]$, as such, expression of MTNR1A was found to be frequently silenced through methylation of CpG islands surrounding the MTNR1A promoter in cases of oral squamous cell carcinoma (OSCC) and other primary cancers [59]. Furthermore, forced expression of MTNR1A in cells led to growth suppression, suggesting that loss of MTNR1A activity plays a role in the pathogenesis of OSCC [59]; similar results have also been found in breast cancer cell lines [60] and in prostate epithelial cells [61]. The antiproliferative effect observed in prostate epithelial cells was demonstrated to be due to MTNR1A-mediated activation of protein kinase A (PKA) and protein kinase $\mathrm{C}$ (PKC) with a subsequent increase in p27 (kip1) gene transcription. The p27 gene encodes for cyclin-dependent kinase inhibitor $1 \mathrm{~B}$, a protein that prevents the activation of cyclin E-CDK2 or cyclin D-CDK4 complexes, thus regulating cell cycle progression. A similar mechanism may be at play in Non-WNT/SHH medulloblastomas.

Another GPCR, the adenosine $\mathrm{A}_{1}$ receptor (ADORA1) has a known role in growth suppression $[62,63]$. In colon cancer cells, adenosine, via ADORA1, induces apoptosis by activating caspases [63]. Additionally, it has been reported that deletion of ADORA1 leads to an increase in glioblastoma tumor growth, however this observed effect was believed to be mediated through tumor-adjacent microglia [64]. ADORA1 was under-expressed in the NonWNT/SHH group (0.088-fold, $\mathrm{p}=0.03)$, again suggesting that loss of ADORA1 activity may play a role in the pathogenesis of a Non-WNT/SHH medulloblastoma tumors, especially those seen in Cluster "E" (Table 1). 
Our data identify GPCRs whose expression is significantly altered in subgroups of medulloblastoma; while many of these alterations reach significant levels, a limitation of our study was the restricted sample size available. To partially alleviate this concern, we worked with the Medulloblastoma Advanced Genomics International Consortium (MAGIC), an international consortium that aims to stratify and characterize medulloblastoma through genomics. Our key findings, specifically the over-expression of LGR5 and GPR64 in the WNT subgroup tumors and F2R and FZD2 in all medulloblastoma, were mirrored in three independent international cohorts of subgrouped medulloblastoma (Figures 3 and 4). Though our data cannot be quantitatively combined with these larger data sets, a qualitative comparison adds substantial confidence and weight to our results.

\section{Conclusions}

In summary, this study has shown that GPCR expression patterns differentiate the WNT and SHH subgroup of tumors. We have identified under-expressed GPCRs that may aid in discerning additional tumor- initiating, or potentiating, pathways at play in medulloblastoma. And importantly, we have pinpointed uniquely over-expressed GPCRs that hold potential as both imaging and therapeutic targets in the WNT and SHH medulloblastoma subgroups.

\section{Additional files}

Additional file 1: Figure S1. GPCR expression patterns delineate distinct groups of medulloblastoma tumors. The heat map represents GPCR expression levels in 41 medulloblastoma tumors compared to normal cerebella. This heat map is the same as is seen in Figure 1, with GPCR names included along the $y$-axis. Control cerebella are outlined in the black box.

Additional file 2: Table S1. Additional medulloblastoma tumor characteristics.

Additional file 3: T3able S2. GPCR expression levels by subgroup compared to normal cerebella.

\section{Competing interests}

The authors declare that they have no conflict of interests.

\section{Authors' contributions}

KLW and MSO co-conceived and led the study. KLW planned and executed the GPCR expression arrays and analyses, aided in the IHC analyses, performed the over-all analyses and drafted the manuscript; EAB performed initial GPCR expression arrays; KNGC developed IHC protocols and provided IHC analysis; PAK provided IHC analysis; BWD provided FISH analysis; QQ performed FISH studies and aided in FISH analysis; WJI provided biospecimens (including RNA) that formed a portion of our medulloblastoma cohort, as well as valuable input regarding interpretation of results and manuscript preparation; TR performed and provided IHC analyses on a subset of our medulloblastoma cohort; MR and MDT provided comparison studies on the expression of select GPCRs in large international cohorts; MR provided valuable input regarding interpretation of results and manuscript preparation; MSO provided financial and technical infrastructure, oversaw the study and significantly aided in drafting the manuscript. All authors read and approved the final manuscript.

\section{Acknowledgements}

This work was supported by grants from St. Baldrick's Foundation and Ride for the Kids (MSO). KLW, member of the University of lowa (UI) Medical Scientist Training Program, was supported by Alex's Lemonade Stand Foundation Pediatric Oncology Student Training program, the National Institute of Neurological Disorders and Stroke of the National Institutes of Health under Award Number T32NS007421, and the Predoctoral Training in the Pharmacological Sciences under Award Number T32GM067795. WJI was supported by the Children's Health Foundation Queensland and The Brainchild Foundation (Brisbane, Australia). A postdoctoral fellowship for MR is funded by the German Cancer Aid/Mildred-Scheel foundation. We would like to thank the Histology Research Laboratory in the Department of Pathology at the University of lowa for their technical expertise.

\section{Author details}

'Department of Pediatrics, Carver College of Medicine, University of lowa, lowa City, IA 2524 JCP, USA. ${ }^{2}$ Neuroscience Graduate Program, Carver College of Medicine, University of lowa, lowa City, IA, USA. ${ }^{3}$ Holden Comprehensive Cancer Center, Carver College of Medicine, University of lowa, lowa City, IA, USA. ${ }^{4}$ Department of Pathology, Carver College of Medicine, University of lowa, 200 Hawkins Drive, lowa City, IA, USA. ${ }^{5}$ Queensland Children's Tumour Bank, Queensland Children's Medical Research Institute, The University of Queensland, Brisbane, QLD, Australia. ${ }^{6}$ Pathology Queensland, Royal Children's Hospital, Brisbane, QLD, Australia. ${ }^{7}$ Arthur and Sonia Labatt Brain Tumour Research Centre, Program in Developmental and Stem Cell Biology, Hospital for Sick Children, University of Toronto, Toronto, ON, Canada.

Received: 19 September 2013 Accepted: 1 October 2013

Published: 10 October 2013

\section{References}

1. Louis DN, Ohgaki H, Wiestler OD: The 2007 WHO classification of tumours of the central nervous system. Acta Neuropathol 2007, 114:97-109. Doi 10.1007/s00401-007-0243-4

2. Mabbott DJ, Spiegler BJ, Greenberg ML, Rutka JT, Hyder DJ, Bouffet E: Serial evaluation of academic and behavioral outcome after treatment with cranial radiation in childhood. J Clin Oncol 2005, 23:2256-2263. Doi:10.1200/jco.2005.01.158

3. Watterson J, Simonton SC, Rorke LB, et al: Fatal brain stem necrosis after standard posterior fossa radiation and aggressive chemotherapy for metastatic medulloblastoma. Cancer 1993, 71:4111-4117.

4. Ellison D: Classifying the medulloblastoma: insights from morphology and molecular genetics. Neuropathol Appl Neurobiol 2002, 28:257-282.

5. Kool M, Korshunov A, Remke $M$, et al: Molecular subgroups of medulloblastoma: an international meta-analysis of transcriptome, genetic aberrations, and clinical data of WNT, SHH, Group 3, and Group 4 medulloblastomas. Acta Neuropathol 2012, 123:473-484. Doi 10.1007/ s00401-012-0958-8.

6. Northcott PA, Dubuc AM, Pfister S, Taylor MD: Molecular subgroups of medulloblastoma. Expert Rev Neurotherapeutics 2012, 12:871-884. Doi 10.1586/ern.12.66.

7. Taylor MD, Northcott PA, Korshunov A, et al: Molecular subgroups of medulloblastoma: the current consensus. Acta Neuropathol 2012, 123:465-472. Doi 10.1007/s00401-011-0922-z.

8. Cho YJ, Tsherniak A, Tamayo P, et al: Integrative genomic analysis of medulloblastoma identifies a molecular subgroup that drives poor clinical outcome. J Clin Oncol 2011, 29:1424-1430. Doi 10.1200/jco.2010.28.5148.

9. Kool M, Koster J, Bunt J, et al: Integrated genomics identifies five medulloblastoma subtypes with distinct genetic profiles, pathway signatures and clinicopathological features. PLoS One 2008, 3:e3088. Doi 10.1371/journal.pone.0003088.

10. Northcott PA, Korshunov A, Witt H, et al: Medulloblastoma comprises four distinct molecular variants. J Clin Oncol 2011, 29:1408-1414. Doi 10.1200/ jco.2009.27.4324.

11. Remke M, Hielscher T, Korshunov A, et al: FSTL5 is a marker of poor prognosis in non-WNT/non-SHH medulloblastoma. J Clin Oncol 2011, 29:3852-3861. Doi 10.1200/jco.2011.36.2798.

12. Remke M, Hielscher T, Northcott PA, et al: Adult medulloblastoma comprises three major molecular variants. J Clin Oncol 2011, 29:2717-2723. Doi 10.1200/jco.2011.34.9373. 
13. Thompson MC, Fuller C, Hogg TL, et al: Genomics identifies medulloblastoma subgroups that are enriched for specific genetic alterations. J Clin Oncol 2006, 24:1924-1931. Doi 10.1200/jco.2005.04.4974.

14. Northcott PA, Jones DT, Kool M, et al: Medulloblastomics: the end of the beginning. Nat Rev Cancer 2012, 12:818-834. Doi 10.1038/nrc3410.

15. Northcott PA, Nakahara $Y$, Wu $X$, et al: Multiple recurrent genetic events converge on control of histone lysine methylation in medulloblastoma. Nat Genet 2009, 41:465-472. Doi 10.1038/ng.336.

16. Parsons DW, Li M, Zhang $X$, et al: The genetic landscape of the childhood cancer medulloblastoma. Science 2011, 331:435-439. Doi 10.1126/ science.1198056.

17. Gibson $\mathrm{P}$, Tong $Y$, Robinson $\mathrm{G}$, et al: Subtypes of medulloblastoma have distinct developmental origins. Nature 2010, 468:1095-1099. Doi 10.1038/ nature09587.

18. Gilbertson RJ, Ellison DW: The origins of medulloblastoma subtypes. Annu Rev Pathol 2008, 3:341-365. Doi 10.1146/annurev. pathmechdis.3.121806.151518

19. Northcott PA, Korshunov A, Pfister SM, Taylor MD: The clinical implications of medulloblastoma subgroups. Nat Rev Neurol 2012, 8:340-351. Doi 10.1038/nrneurol.2012.78

20. Robarge KD, Brunton SA, Castanedo GM: GDC-0449-a potent inhibitor of the hedgehog pathway. Bioorg Med Chem Lett 2009, 19:5576-5581. Doi 10.1016/j.bmcl.2009.08.049.

21. Rudin CM, Hann CL, Laterra J, et al: Treatment of medulloblastoma with hedgehog pathway inhibitor GDC-0449. N Engl J Med 2009, 361:1173-1178. Doi 10.1056/NEJMoa0902903.

22. Wu J, Xie N, Zhao X, Nice EC, Huang C: Dissection of aberrant GPCR signaling in tumorigenesis-a systems biology approach. Cancer Genomics Proteomics 2012, 9:37-50.

23. Marti-Solano M, Guixa-Gonzalez R, Sanz F, Pastor M, Selent J: Novel insights into biased agonism at $\mathrm{G}$ protein-coupled receptors and their potential for drug design. Curr Pharm Des 2013.

24. Khanna G, Bushnell D, O'Dorisio MS: Utility of radiolabeled somatostatin receptor analogues for staging/restaging and treatment of somatostatin receptor-positive pediatric tumors. Oncologist 2008, 13:382-389. Doi 13/4/382.

25. Khanna G, O'Dorisio MS, Menda Y, et al: Somatostatin receptor scintigraphy in surveillance of pediatric brain malignancies. Pediatr Blood Cancer 2008, 50:561-566. Doi 10.1002/pbc.21194.

26. Beutler $D$, Avoledo $P$, Reubi JC: Three-year recurrence-free survival in a patient with recurrent medulloblastoma after resection, high-dose chemotherapy, and intrathecal Yttrium-90-labeled DOTA0-D-Phe1-Tyr3octreotide radiopeptide brachytherapy. Cancer 2005, 103:869-873. Doi 10.1002/cncr.20822.

27. Menda Y, O'Dorisio MS, Kao S, et al: Phase I trial of 90Y-DOTATOC therapy in children and young adults with refractory solid tumors that express somatostatin receptors. J NuCl Med 2010, 51:1524-1531. Doi jnumed.110.075226.

28. O'Dorisio MS, Khanna G, Bushnell D: Combining anatomic and molecularly targeted imaging in the diagnosis and surveillance of embryonal tumors of the nervous and endocrine systems in children. Cancer Metastasis Rev 2008, 27:665-677. Doi 10.1007/s10555-008-9153-8.

29. Fredriksson $\mathrm{R}$, Schioth $\mathrm{HB}$ : The repertoire of G-protein-coupled receptors in fully sequenced genomes. Mol Pharmacol 2005, 67:1414-1425. Doi 10.1124/mol.104.009001.

30. Maurel B, Le Digarcher A, Dantec C, Journot L: Genome-wide profiling of G protein-coupled receptors in cerebellar granule neurons using highthroughput, real-time PCR. BMC Genomics 2011, 12:241. Doi 10.1186/14712164-12-241.

31. Mestdagh $P$, Van Vlierberghe $P$, De Weer $A$, et al: A novel and universal method for microRNA RT-qPCR data normalization. Genome biology 2009, 10:R64. Doi 10.1186/gb-2009-10-6-r64.

32. Ellison DW, Dalton J, Kocak M, et al: Medulloblastoma: clinicopathological correlates of $\mathrm{SHH}, \mathrm{WNT}$, and non-SHH/WNT molecular subgroups. Acta Neuropathol 2011, 121:381-396. Doi 10.1007/s00401-011-0800-8.

33. Jung B, Ahmad N: Melatonin in cancer management: progress and promise. Cancer Res 2006, 66:9789-9793. Doi 10.1158/0008-5472.can-06-1776.

34. Castellone MD, Teramoto H, Williams BO, Druey KM, Gutkind JS: Prostaglandin E2 promotes colon cancer cell growth through a Gs-axin-beta-catenin signaling axis. Science 2005, 310(5753):1504-1510.
35. Choy $H$, Milas L: Enhancing radiotherapy with cyclooxygenase-2 enzyme inhibitors: a rational advance? J Natl Cancer Inst 2003, 95:1440-1452.

36. Remke M, Hering E, Gerber NU, et al: Somatostatin receptor subtype 2 (sst) is a potential prognostic marker and a therapeutic target in medulloblastoma. Childs Nerv Syst 2013. Doi 10.1007/s00381-013-2142-4.

37. Fruhwald MC, O'Dorisio MS, Pietsch T, Reubi JC: High expression of somatostatin receptor subtype 2 (sst2) in medulloblastoma: implications for diagnosis and therapy. Pediatr Res 1999, 45:697-708.

38. Fruhwald MC, Rickert CH, O'Dorisio MS: Somatostatin receptor subtype 2 is expressed by supratentorial primitive neuroectodermal tumors of childhood and can be targeted for somatostatin receptor imaging. Clin Cancer Res 2004, 10:2997-3006.

39. Innamorati G, Valenti M, Giovinazzo F, Carbonare L, Parenti M, Bassi C: Molecular approaches to target GPCRs in cancer therapy. Pharmaceuticals 2011, 4:567-589. Doi 10.3390/ph4040567.

40. Bajetta E, Catena L, Valente M, Bianco N, Bellomo F, Bombardieri E: New perspectives in the treatment of neuroendocrine tumours. Anticancer Res 2012, 32:4193-4200.

41. Ogawa K, Yoshioka Y, Isohashi F, Seo Y, Yoshida K, Yamazaki H: Radiotherapy targeting cancer stem cells: current views and future perspectives. Anticancer Res 2013, 33:747-754.

42. Carmon KS, Lin Q, Gong X, Thomas A, Liu Q: LGR5 interacts and cointernalizes with Wnt receptors to modulate Wnt/beta-catenin signaling. Mol Cell Biol 2012, 32:2054-2064. Doi 10.1128/mcb.00272-12.

43. Al-Kharusi MR, Smartt HJ, Greenhough A, et al: LGR5 promotes survival in human colorectal adenoma cells and is upregulated by PGE2: implications for targeting adenoma stem cells with NSAIDs. Carcinogenesis 2013, 34:1150-1157. Doi 10.1093/carcin/bgt020.

44. Kemper K, Prasetyanti PR, De Lau W, Rodermond H, Clevers H, Medema JP: Monoclonal antibodies against Lgr5 identify human colorectal cancer stem cells. Stem Cells 2012, 30:2378-2386. Doi 10.1002/stem.1233.

45. Carmon KS, Gong X, Lin Q, Thomas A, Liu Q: R-spondins function as ligands of the orphan receptors LGR4 and LGR5 to regulate Wnt/betacatenin signaling. Proc Natl Acad Sci USA 2011, 108:11452-11457. Doi 10.1073/pnas.1106083108.

46. de Lau WB, Snel B, Clevers HC: The R-spondin protein family. Genome biology 2012, 13:242. Doi 10.1186/gb-2012-13-3-242.

47. Banks WA: Characteristics of compounds that cross the blood-brain barrier. BMC Neurol 2009, 9(Suppl 1):S3. Doi 10.1186/1471-2377-9-s1-s3.

48. Seelig A: The role of size and charge for blood-brain barrier permeation of drugs and fatty acids. J Mol Neurosci 2007, 33:32-41.

49. Richter GH, Fasan A, Hauer K, et al: G-Protein coupled receptor 64 promotes invasiveness and metastasis in Ewing sarcomas through PGF and MMP1. J Pathol 2013, 230:70-81. Doi 10.1002/path.4170.

50. Fulton AM, Ma X, Kundu N: Targeting prostaglandin E EP receptors to inhibit metastasis. Cancer Res 2006, 66:9794-9797. Doi 10.1158/0008-5472. can-06-2067.

51. Loh JK, Hwang SL, Lieu AS, Huang TY, Howng SL: The alteration of prostaglandin E2 levels in patients with brain tumors before and after tumor removal. J Neurooncol 2002, 57:147-150.

52. Wang D, Dubois RN: Prostaglandins and cancer. Gut 2006, 55:115-122. Doi 10.1136/gut.2004.047100.

53. Baryawno N, Sveinbjornsson B, Eksborg S, et al: Tumor-growth-promoting cyclooxygenase-2 prostaglandin E2 pathway provides medulloblastoma therapeutic targets. Neuro-oncology 2008, 10:661-674. Doi 10.1215/ 15228517-2008-035.

54. Boyd MJ, Berthelette C, Chiasson JF: A novel series of potent and selective $\mathrm{EP}(4)$ receptor ligands: facile modulation of agonism and antagonism Bioorg Med Chem Lett 2011, 21:484-487. Doi 10.1016/j.bmcl.2010.10.106.

55. Goessling W, North TE, Loewer S, et al: Genetic interaction of PGE2 and Wnt signaling regulates developmental specification of stem cells and regeneration. Cell 2009, 136:1136-1147. Doi 10.1016/j.cell.2009.01.015.

56. Katoh $Y$, Katoh M: Hedgehog target genes: mechanisms of carcinogenesis induced by aberrant hedgehog signaling activation. Curr Mol Med 2009, 9:873-886.

57. Martin V, Herrera F, Carrera-Gonzalez P, et al: Intracellular signaling pathways involved in the cell growth inhibition of glioma cells by melatonin. Cancer Res 2006, 66:1081-1088. Doi 10.1158/0008-5472.can-05-2354.

58. Vijaya L, Thomas CR Jr, Reiter RJ, Herman TS, et al: Melatonin: from basic research to cancer treatment clinics. J Clin Oncol 2002, 20:2575-2601. 
59. Nakamura E, Kozaki K, Tsuda $\mathrm{H}$, et al: Frequent silencing of a putative tumor suppressor gene melatonin receptor $1 \mathrm{~A}$ (MTNR1A) in oral squamous-cell carcinoma. Cancer Sci 2008, 99:1390-1400. Doi 10.1111/ j.1349-7006.2008.00838.x.

60. Yuan L, Collins AR, Dai J, Dubocovich ML, Hill SM: MT(1) melatonin receptor overexpression enhances the growth suppressive effect of melatonin in human breast cancer cells. Mol Cell Endocrinol 2002, 192:147-156.

61. Tam CW, Chan KW, Liu WW, Pang B, Yao KM, Shiu SY: Melatonin as a negative mitogenic hormonal regulator of human prostate epithelial cell growth: potential mechanisms and clinical significance. J Pineal Res 2008, 45:403-412. Doi 10.1111/j.1600-079X.2008.00608.x.

62. Merighi S, Simioni $\mathrm{C}$, Gessi $\mathrm{S}$, et al: $\mathrm{A}(2 \mathrm{~B})$ and $\mathrm{A}(3)$ adenosine receptors modulate vascular endothelial growth factor and interleukin-8 expression in human melanoma cells treated with etoposide and doxorubicin. Neoplasia 2009, 11:1064-1073.

63. Saito M, Yaguchi T, Yasuda Y, Nakano T, Nishizaki T: Adenosine suppresses CW2 human colonic cancer growth by inducing apoptosis via $A(1)$ adenosine receptors. Cancer Lett 2010, 290:211-215. Doi 10.1016/j. canlet.2009.09.011.

64. Fishman P, Bar-Yehuda S, Synowitz M, et al: Adenosine receptors and cancer. Handbook Exp Pharmacol 2009:399-441. Doi 10.1007/978-3-54089615-9_14.

doi:10.1186/2051-5960-1-66

Cite this article as: Whittier et al:: G-protein coupled receptor expression patterns delineate medulloblastoma subgroups. Acta Neuropathologica Communications 2013 1:66

\section{Submit your next manuscript to BioMed Central and take full advantage of:}

- Convenient online submission

- Thorough peer review

- No space constraints or color figure charges

- Immediate publication on acceptance

- Inclusion in PubMed, CAS, Scopus and Google Scholar

- Research which is freely available for redistribution 